\title{
Transsaccadic memory of position and form
}

\author{
Heiner Deubel ${ }^{1, *}$, Werner X. Schneider ${ }^{2}$ and Bruce Bridgeman ${ }^{3}$ \\ ${ }^{1}$ Experimental Psychology, Ludwig-Maximilians-Universität, Leopoldstrasse 13, 80802 Munich, Germany \\ ${ }^{2}$ Experimental Psychology, Ludwig-Maximilians-Universität, Leopoldstrasse 13, 80802 Munich, Germany \\ ${ }^{3}$ Department of Psychology, University of California, Social Sciences 2, Santa Cruz, CA 95064, USA
}

\begin{abstract}
Why and how people perceive the visual world as continuous and stable, despite the gross changes of its retinal projection that occur with each saccade, is one of the classic problems in perception. In the present paper, we argue that an important factor of visual stability and transsaccadic perception is formed by the reafferent visual information, i.e., the visual display that is present when the eyes land. After a review of some of the relevant theoretical, behavioural and physiological research on space constancy, saccadic suppression and transsaccadic memory, three experiments are presented. In a first experiment, we study the effect of an extended horizontal bar covering the target area for a short period after the saccade on saccadic suppression of image displacement. The results show that the bar acts just like a temporary blanking of the saccade target, leading to a strong reduction of saccadic suppression. In the second experiment, we show that any object that is present immediately after the saccade can establish a spatial reference, even if it is dissimilar to the saccade target. In a third experiment we study, with a similar approach, the effect of blanking and postsaccadic information on transsaccadic integration of form information. The data demonstrate that a localized postsaccadic object tends to replace the content of transsaccadic memory.
\end{abstract}

Keywords: Saccades; Saccadic suppression; Transsaccadic memory; Visual stability; Space constancy; Reference object; Blanking

\section{Theoretical background}

\section{Introduction}

Visual information exists all around us, but physiological constraints prevent us from seeing it all at once. When the eye fixates an area, high resolution is limited to a narrow region around the central fovea. Therefore, saccades are required that bring different regions of the world into view. However, such eye movements induce several problems that

\footnotetext{
* Correspondence to: H. Deubel, Experimental Psychology, Ludwig-Maximilians-Universität, Leopoldstrasse 13, 80802 Munich, Germany. Tel.: +49-89-2180-5282; Fax: +49-89-2180-5211; E-mail: deubel@psy.uni-muenchen. de
}

the perceptual system must solve. A first problem is that, because of the high eye velocity during a saccade, the visual input is reduced or eliminated during the time of a saccade. Nevertheless, we do not perceive repetitive 'wipe-outs' of the visual information. Second, the images of the objects in the world drastically change their retinal positions during each saccade. Nevertheless, we do not remain disoriented with each saccade. Space constancy is normally perfect, the world does not appear to jump in the slightest when the eye moves. By contrast, a comparable retinal image motion, produced externally by having the observer tap on his eyeball, produces an alarming percept of instability.

Why and how people perceive the visual world as continuous and stable, despite the gross changes of its retinal projection that occur with each saccade, is certainly one of the classic problems in perception. In 
the present paper, we want to argue that an important factor of visual stability and transsaccadic perception is formed by the reafferent visual information, i.e., the visual display that is present when the eyes land. But before we present our own data, we will review some of the relevant theoretical, behavioural and physiological research on space constancy, saccadic suppression and transsaccadic memory.

\section{Space constancy and the role of efference copies during saccadic eye movements}

One of the first accounts of the problem of space constancy was provided by Helmholtz (see Helmholtz, 1963). He assumed that retinal image motion due to eye or body movements is sensed, but not perceived. Constancy of visual direction is maintained by combining the image motion and the "effort of will involved in trying to alter the effort of will". Closely related, more modern attempts to account for space constancy were mainly cancellation theories, in which the sensory effects of an eye movement are compensated by a simultaneous, equal and opposite extraretinal signal about the position of the eyes in the orbit (Sperry, 1950; von Holst and Mittelstaedt, 1954). The retinal and extraretinal signals cancel each other somewhere in the brain, resulting in a space-constant representation of visual space. In these theories an oculomotor efference copy, proprioception, or some combination of both subtracts from the disturbing effects of a displaced retinal image following a saccade.

Cancellation theories cannot support space constancy unaided, however, because the extraretinal signals are not exact copies of the actual eye movement. First, their gain (ratio of extraretinal signal to actual eye movement) is usually less than one (Grüsser et al., 1987), so they are too small to afford complete compensation. Also dynamically, extraretinal signals of eye position are far from perfect. Direction constancy for flashed stimuli in darkness is grossly disturbed in the vicinity of the saccade. Leonard Matin and colleagues (e.g., Matin, 1972) and Bischof and Kramer (1968) were among the first to study errors in the localization of flashed objects around the time of saccadic eye movements. These and a large number of subsequent studies (e.g., Honda, 1989; Schlag and Schlag-Rey, 1995) analyzed the perception of short localized flashes before, during or after a saccade. The general finding was that these stimuli are systematically mislocalized. Mislocalization starts about $100 \mathrm{~ms}$ before the eyes begin to move, where flashes have a tendency to be seen as displaced in the direction of the saccade. Perceived displacement reaches a maximum around the time of the onset of the saccadic movement. These perceptual displacements are presumably a reflection of the mechanisms that compensate for the actual shift in retinal position brought about by the movement of the eye.

In a more detailed account of saccadic mislocalization, recent research has demonstrated that the mislocalization of flashes before and during saccades is not spatially homogeneous. Ross et al. (1997) showed that targets are not simply perceived as displaced in the direction of the saccade. Rather, objects that are closer than the saccade target are perceived as being displaced into the direction of the saccade, and those that are further away than the target are perceived as closer. In other words, targets flashed before and during saccades tend to converge towards the saccade target which results in an apparent 'compression' of the visual world around the saccade target. This compression can even be perceived for natural images presented shortly during the saccade (Ross et al., 1997). Lappe et al. (2000) recently studied saccade-induced mislocalization under various conditions. Interestingly, they found that compression of visual space only occurs when visual references were available after the saccade. Other studies have shown that visual references also modify the gain of the presaccadic mislocalization (Honda, 1999).

Thus it seems that extraretinal information about eye position is notoriously imprecise, statically and dynamically. However, even a small error of the extraretinal signal should result in a disturbance of constancy. One compelling solution to this problem is that the visual system has the built-in assumption that the world as a whole does not change during an eye movement. A mechanism that becomes important here is saccadic suppression; indeed, it has been suggested that saccadic suppression 'bridges the errors' that remain due to the imperfect cancellation mechanism (Bridgeman et al., 1994). Therefore, let us next look at saccadic suppression. 


\section{Saccadic suppression}

Saccadic suppression is a reduction of the visual sensitivity to events occurring before, during, and immediately after saccadic eye movements. Two separate types of saccadic suppression should be distinguished. First, there are many studies on the visual sensitivity to short flashes presented around the time of the saccade (for a review, see, e.g., Matin, 1974). Typically, these studies have reported a moderate threshold elevation (two to threefold) for detecting spots of light flashed briefly during saccades. Other researchers used gratings that were briefly presented during saccades. Their results demonstrate that saccadic suppression is strongest for the low spatial frequencies (below 1 cycle per degree), while higher spatial frequencies remain largely unaffected (Wolf et al., 1978, 1980; Burr et al., 1994). This selectivity of suppression to the magnocellular pathway strongly suggests that this type of suppression is specific to motion signals (see, e.g., Ross et al., 2001).

The second type of saccadic suppression, more relevant in the context discussed here, concerns the detection of image displacement that occurs during saccadic eye movements. During fixation, the sensitive motion detectors of the visual system allow to perfectly perceive even very small displacements of visual objects. Due to the high retinal velocity during a saccade, however, these signals are basically 'wiped-out' with each eye movement. This leads to a strong reduction in sensitivity (by three to four log units) for detecting displacements during saccades (Bridgeman et al., 1975). Magnocellular pathways are also implicated in saccadic suppression of displacement (Bridgeman and Macknik, 1995).

The threshold increase for image jumps during saccades is much larger than the threshold increase for other changes such as brightness increments. In the context of recent work, saccadic suppression of image displacement can be interpreted as a special case of 'change blindness' (Rensink et al., 1997; Rensink, 2002), the inability of human subjects to identify changes that take place in a visual scene from one fixation to the next, or even within a single fixation if a blank interval prevents direct motion perception.

Without the direct evidence for a target jump from motion detectors, detection of intrasaccadic image displacement requires the comparison of pre- and postsaccadic target locations. Saccadic suppression of image displacement therefore seems to imply either that the required precise comparison is normally not performed, or that transsaccadic memory about the location of objects is not available to the visual system, or is very poor. Indeed, Bridgeman et al. (1994) in their theoretical account of visual stability assumed that there is no need for transsaccadic memory of object positions, rather, the spatial positions are calculated anew after each saccade. This raises the question of the nature of transsaccadic memory, and of what and how much information is contained in this store.

\section{Transsaccadic memory}

At the core of most accounts of visual stability is the assumption that some information is stored from one fixation to the next. In its extreme version, this assumption would suggest that presaccadic and postsaccadic information are integrated into a very detailed, high-capacity spatial buffer that combines information from one fixation to the next. Such a mechanism of spatiotopic superposition has indeed been proposed by several authors (e.g., McConkie and Rayner, 1976; Wolf et al., 1980; Jonides et al., 1982; Breitmeyer, 1984). However, despite the intuitive appeal of this hypothesis, ample empirical evidence has demonstrated that it is probably wrong. Several investigators have found that subjects are unable to fuse pre- and postsaccadic patterns in successive fixations to obtain an integrated composite pattern (e.g., Irwin, 1983; O'Regan and Levy-Schoen, 1983; Rayner and Pollatsek, 1983; for a comprehensive overview see also Irwin, 1993a). Moreover, it has been shown that changing the visual characteristics of words and pictures, such as object size or letter case, has no disruptive effect on word or picture naming (e.g., McConkie and Zola, 1979; Rayner et al., 1980). More importantly, it has also no consequences on eye movements in reading. McConkie and Zola (1979) have used cAsE AlTeRnAtIoNs from one fixation to another and found that readers did not notice these changes, and that they did not affect eye movements.

But if the successive 'snapshots' of the world are not fused into a transsaccadic buffer, how can 
the visual system then produce a stable and continuous representation of a scene? The current assumption is that transsaccadic memory exists but is less image-like in form, containing instead more abstract representations of the information present in each fixation. Rayner et al. (1980) found that a word presented in one fixation speeded the naming of the word in the next fixation, irrespective of an intrasaccadic change of the letter case. Pollatsek et al. (1984) demonstrated that visual and conceptual similarity facilitated the identification of objects across saccades, regardless of changes in object size. So, it seems that abstract visual features and identity codes are combined across fixations. More recent work has also emphasized that while memory for absolute spatial positions across saccades is poor, relational information is well retained from one fixation to the next (e.g., Carlson-Radvansky, 1999; Verfaillie and De Graef, 2000).

In addition to the question of the level of the transsaccadic representations, another important aspect concerns the amount of information that is stored across the saccade. Irwin, 1992 (see also below) found that subjects could remember between three and four letters across an eye movement. Memory capacity for visual elements was estimated to be between three and six elements (Irwin, 1993b).

In summary, there is now agreement that information integration across saccades is carried out at an abstract level. Transsaccadic memory seems to be an undetailed, limited-capacity memory. It is relatively long-lasting (more than several seconds, Irwin, 1991) and is not strictly tied to spatial position. When transsaccadic memory capacity is measured in number of items, it is estimated that three to six memory items survive the saccade. In all these properties transsaccadic memory is similar to, if not identical with, visual short-term memory (Irwin, 1991). For recent and comprehensive overviews of further various aspects of transsaccadic memory see, e.g., Rayner (1998) and De Graef and Verfaillie (2001, 2002).

\section{Is there a special role for the saccade target?}

A number of theories have emphasized a special role of the processing of the saccade target for perceptual stability and transsaccadic memory. Deubel et al. (1984) were probably the first to propose that a transsaccadic memory representation of the saccade target may serve to relocate visual objects across saccades. In more recent work, we (Deubel and Schneider, 1994; Deubel et al., 1996, 1998) developed a 'reference object theory' that assumes that pre- and postsaccadic visual 'snapshots' are linked by means of the saccade target which is assumed by the visual system as being stable. In a very similar theoretical approach, the 'saccade target theory' (McConkie and Currie, 1996; Currie et al., 2000) also assigns a privileged status to the object that constitutes the target for the saccade. Both theories assume that with each new fixation the visual system runs through a sequence of processing steps which starts with the selection of one object as the target for the next saccade. Particular features about the saccade target are selected and stored in a transsaccadic memory to facilitate the re-identification of the target at the start of the next fixation. Then the saccade is executed that brings the target object into central vision. After the eye has landed, the visual system searches for the critical target features within a limited region around the landing site. If the target object is found, the relationship between its retinal location and its mental representation is compared in order to coordinate these two types of information. If the postsaccadic target localization fails (e.g., because the intrasaccadic target shift was too large), however, the assumption of visual stability is abandoned. As a consequence, a target displacement is perceived.

There is some empirical evidence that supports the assumption of a preferential transsaccadic processing of the saccade target. In a study by Irwin (1992) subjects were presented an array of letters while they fixated a central fixation point; the onset of a peripheral stimulus indicated the saccade target. The letters disappeared with the saccade, following the eye movement, the subjects were required to report one of the letters in a partial report paradigm. Irwin found that subjects could remember only 3-4 letters, and that report of the letters near the saccade target was much more accurate than of the other letters in the array. This suggests that information near the saccade target is more likely to be encoded in transsaccadic memory than information from more distant locations. McConkie and Currie (1996) used full-colour pictures of natural scenes which changed 
during the saccade. In their Experiment 2, the scene expanded or contracted during the eye movement. McConkie and Currie found that the detectability of these image changes was a direct function of the displacement size at the location where the eyes landed, confirming the importance of the local region around the saccade target. Finally, Currie et al. (2000), also using full-colour pictures of natural scenes, studied the detectability of intrasaccadic displacements of objects in the display. They found that displacements of the saccade target object were much easier to detect than displacements of the background (with the saccade target object remaining stationary).

A strong version of the 'saccade target theory' would predict that only information about the saccade target would be stored across the saccade, while intrasaccadic changes of other objects would remain imperceptible. There is clear empirical evidence against this conjecture, however. So, Deubel et al. (1998) presented a simple configuration of a saccade target and a nearby distractor, one of both objects was displaced by a small amount during the saccade. The data revealed no systematic preference for the displacement of the saccade target to be detected. Verfaillie and De Graef (2000) made subjects saccade from one biological motion walker at the fixation position to another in the visual periphery. During the saccade, either the walker at the launch site or the walker which was the saccade target changed in depth orientation or in location. The results show that change detection for the walker at the saccade target was not more accurate than for the walker at the presaccadic fixation.

An important factor to consider in this context is visual attention. A number of investigators have shown that attention movements obligatorily precede saccadic eye movements, leading to a selective improvement of the detection and identification of items presented at the saccade target location, and to a deterioration of performance at other stimulus locations (e.g., Hoffman and Subramaniam, 1995; Kowler et al., 1995; Deubel and Schneider, 1996). This is so even if subjects are instructed to attend to a location other than the saccade target. Irwin and Gordon (1998) manipulated attention in a transsaccadic letter recognition task. Subjects were encouraged to attend to one region in a display while they moved their eyes either to the region they were attending or to another region. The results show that accuracy was high at positions that subjects were asked to attend to, but it was about equally high for positions close to the saccade target, even if the subjects were asked to attend elsewhere. So, the effect of making a saccade to a location produced as much benefit as biasing the subject to attend to a location. Irwin and Gordon (1998) also found, as did Irwin (1992), that subjects were able to remember about four items of the letter display, supporting the argument that the capacity of transsaccadic memory is approximately four objects.

The findings that attention precedes eye movements and that transsaccadic memory capacity is about four items combined may solve the seeming contradictions in the research described above. It has been frequently suggested that the encoding of a visual stimulus in working memory requires selective attention (e.g., Schneider, 1999). In scenes consisting of only two objects like in the experiments of Deubel et al. (1996) and Verfaillie and De Graef (2000) it is likely that both objects are attended before the saccade, so both stimuli are encoded with sufficient accuracy in transsaccadic memory. The more complex stimuli of Irwin (1992), McConkie and Currie (1996) and Currie et al. (2000), however, exceed the capacity of the visual memory; hence only a selected subset of the stimuli, preferentially around the saccade target area, will be still in memory for further processing after the saccade.

\section{The role of reafferent information: the blanking effect}

The previous paragraphs discussed the question of what is contained in transsaccadic memory, and why it is encoded. However, an important, but often neglected aspect of memory performance arises only at the moment when memory is probed, which is here when the saccade lands. Then, a comparison has to take place of the contents of transsaccadic memory and the actual reafferent visual information. The question arises how this comparison works and to what extent the stored information content may be affected, and possibly, overwritten, by the new retinal information.

We here propose that the effect of the postsaccadic information on the contents of transsaccadic memory is indeed an important factor for both 
transsaccadic memory and perceived visual stability. Evidence for our conjecture comes from our experiments on saccadic suppression of image displacement with simple targets (Deubel and Schneider, 1994; Deubel et al., 1996). In these experiments, we demonstrated that saccadic suppression largely disappears with a stunningly simple manipulation, namely by blanking the target with saccade onset and restoring it only 50-300 ms after the eyes stop at the end of a saccade; we called this effect the 'blanking effect'. The blanking effect occurs even for targets in darkness, meaning that displacement detection under this condition relies on extraretinal signals rather than on retinal information from the structured environment. It has been argued that saccadic suppression of image displacement implies that transsaccadic information about spatial positions is poor. However, the considerable accuracy with which subjects can judge transsaccadic displacements in the 'blanking' condition clearly requires both the maintenance of high-quality information about presaccadic target position across the saccade, and a precise extraretinal signal! Thus, it follows from our findings that precise information about the presaccadic target position and a precise extraretinal signal are indeed available for stimulus localizations after the saccade, but they ordinarily are not used in perception. We have suggested that this is because the visual system assumes, as a null-hypothesis, the stability of any object that is continuously available both before and after the saccade. Only a very large discrepancy between eye movement magnitude and image position is able to break this assumption. This assumption is also broken, however, when the presaccadic object is not present immediately after the saccade. Only under this condition are precise transsaccadic information and extraretinal signals used to achieve displacement detection. Because of its strong effect in unveiling information available transsaccadically, target blanking presents a tool for studying visual stability and the nature of spatial information transferred across the saccade.

While the absence of a postsaccadic target eliminates saccadic suppression, its presence largely determines whether other stimuli in the field are seen as stable. We demonstrated this in the experiments with two stimuli (a target and a distractor) already described above (Deubel et al., 1998). One of the manipulations in these experiments included a postsaccadic blanking of one of the stimuli, while the other stimulus was displaced during the saccade. Even when the postsaccadic blank was very short (e.g., $50 \mathrm{~ms}$ ), the blanked object was invariably perceived as moving across the saccade, while the moved (but continuously present) object was perceived as stable. The fact that this striking illusion even occurred for object displacements of up to half of the size of the saccade illustrates that under this condition perceptual stability is determined not by extraretinal signals but by the object that is found when the eyes land.

This research showed that an object is perceived as stable if it is visible at the end of the eye movement. If the target is blanked even for only $50 \mathrm{~ms}$ after the saccade, the visual system fails to find it and the assumption of target stability is broken. In natural visual environments, the stabilization system works well because objects almost never disappear across saccades. However, objects of fixation frequently become obscured briefly by other objects, such as blowing leaves or snow. If the period of occlusion is brief, the object is still perceived, but it is perceived to be behind the occluding object. The effect has been called amodal completion by Michotte (1963), who has described many of its properties. Amodal completion is so effective that we are normally unaware that an object of attention has been briefly obscured. The question arises whether the space constancy system can also tolerate such temporary absence of a saccade target. In other words, is amodal completion as effective as the continuous presence of the target for maintaining constancy? For this purpose, in Experiment 1 the target region is covered, for $250 \mathrm{~ms}$ after a saccade, with a bar that provides no information about target location. If the space constancy system is capable of amodal completion, as in perception of occluded objects, this bar should have no effect on the perceived continuity of target location, and detection of an intrasaccadic displacement should be poor. If space constancy requires actual physical presence of the target, however, detection of the displacement should be good, just as with the blanking effect when the target is extinguished and later reappears. In Experiment 2 we provide a pattern during the blanking interval that has no configurational similarity or spatial overlap 
with the saccade target, but specifies the potential location of the target. The experiment investigates whether any object that is present immediately after the saccade can establish a spatial reference, even if it is dissimilar to the saccade target. Experiment 3 studies, with a similar approach, the effect of blanking and postsaccadic information on transsaccadic integration of form information.

\section{Experiments}

\section{Experiment 1: occluding the stimulus}

Methods

Seven paid subjects participated in this experiment. They were naive with respect to the object of the study, but were experienced with the equipment from other eye-movement-related tasks, and had normal visual acuity. Each subject performed at least three separate sessions in each paradigm. For each experiment, the results shown are based on 100-200 trials per condition from each subject. Stimuli were presented on a $21^{\prime \prime}$ video monitor at a frame rate of $100 \mathrm{~Hz}$. Screen background luminance was 2.2 $\mathrm{cd} / \mathrm{m}^{2}$; the luminance of the saccade target and of other stimuli was $25 \mathrm{~cd} / \mathrm{m}^{2}$. The subjects viewed the screen binocularly from a distance of $80 \mathrm{~cm}$. Head movements were restricted by a bite board and a forehead rest. Eye movements were measured with an SRI Generation 5.5 Purkinje-image eyetracker (Crane and Steele, 1985) and sampled at a rate of $500 \mathrm{~Hz}$. Further details of computer control, calibration and triggering are given in Deubel et al. (1996).

The visual target consisted of a small white cross subtending a visual angle of $0.2^{\circ}$. The subject's task was to maintain fixation on the target, and to track it with a saccade if it jumped across the visual field. At the start of each trial the target jumped left or right $6^{\circ}$ or $8^{\circ}$ to elicit a saccade. The two amplitudes and directions were randomized and equally probable to minimize anticipation and adaptation effects. Saccades beginning earlier than $140 \mathrm{~ms}$ or later than $400 \mathrm{~ms}$ after the target step were discarded. The computer triggered a second displacement when it detected the saccade elicited by the first target step (see Fig. 1A). The size of the second target displacement was $0.6^{\circ}$, and it was elicited either into the same or into the opposite direction of the first step. At the end of each trial, in a two-alternative forcedchoice procedure, the subject's task was to report the direction of the second target shift with respect to the direction of the primary saccade ('forward' vs. 'backward').

The different sequences of stimulus presentations of this experiment are sketched in Fig. 1A. Four different experimental conditions were included. In a first control condition, the target was displaced during the primary saccade, but no blanking occurred ('No blank' condition). In a second condition, the primary saccade triggered a blanking of the target which lasted $250 \mathrm{~ms}$ ('blank' condition); then the displaced target reappeared. A blanking time of 250 ms was found to fully elicit the blanking effect in a previous study (Deubel et al., 1996). In the third condition, a horizontal bar, $28^{\circ}$ long, extending across the entire width of the screen, appeared during the $250 \mathrm{~ms}$ period ('Occluding bar' condition). While the target was not present, from the detection of the saccade until $250 \mathrm{~ms}$ thereafter, the bar was displayed at the horizontal level of the target, covering both the previous target position and the various possible post-blanking target positions. Finally, in an additional control condition ('Masking bars' condition), two bars identical to the occluding bar were presented above and below the target region. Like the single long bar, they provided no information about the eventual (horizontal) position of the target, but this configuration did not cover the position of the target. Here the target was not occluded behind another object but clearly had disappeared during the blanking interval. The bars were each $28^{\circ}$ long and $0.56^{\circ}$ high. They were displayed only during the 250 ms blank period.

\section{Results}

Fig. 1B gives the discrimination performance in the four experimental conditions as percent correct; chance level is $50 \%$. First, the data replicate the blanking effect described in our previous work: while the discrimination of intrasaccadic displacements is close to chance level for the non-blanked condition in which the target is continuously present, performance is dramatically enhanced by the introduction of the $250 \mathrm{~ms}$ blanking period, reaching about $92 \%$ 


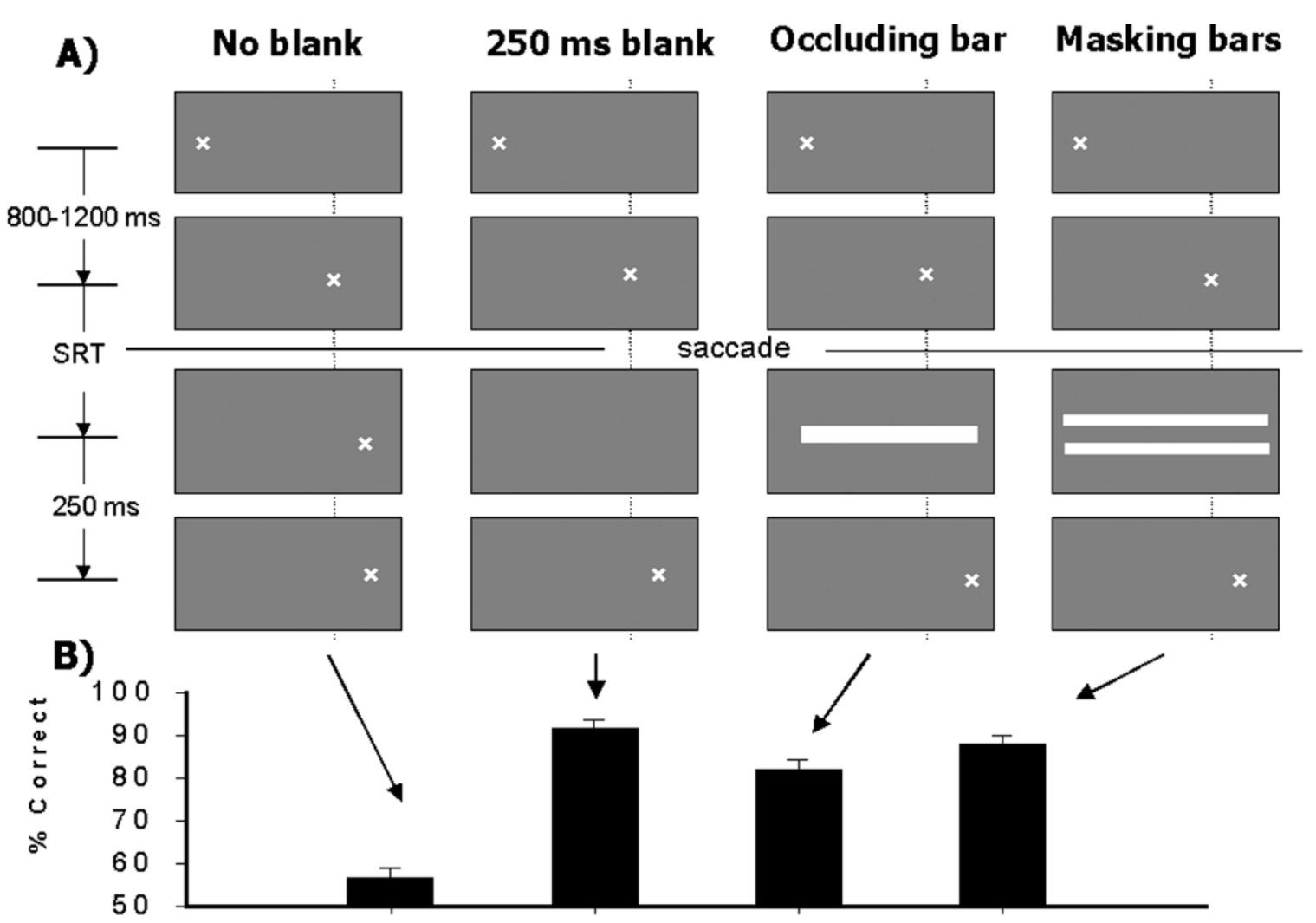

Fig. 1. (A) Experimental conditions in Experiment 1. The open rectangles show the appearance of the screen in successive time intervals. The small ' $x$ ' represents the saccade target. SRT $=$ saccadic reaction time. First column from left: 'no blank' control condition. Second column: condition with $250 \mathrm{~ms}$ postsaccadic target blanking. Third column: stimulus sequence with a long bar covering the target area during the blanking interval. Rightmost column: comparison condition with two bars that do not obscure the location of the saccade target. (B) The data show percent correct displacement discrimination with no blank, a $250 \mathrm{~ms}$ blanking, with a long occluding bar present throughout the $250 \mathrm{~ms}$ blank, and with two long bars present throughout the blank interval. Error bars indicate between-subject standard errors.

correct. This means that subjects can correctly report the target displacements when the target is absent for a short period immediately after the saccade. Second, the data show that detectability of a target jump is also improved considerably (with respect to the 'No blank' condition) when the target's possible post-blanking positions are occluded by the long bar. In this case performance is intermediate between the blanking and the non-blanking detectabilities. The target displacement detectability with the occluding bar is significantly better than in the no-blank condition $(t(6)=4.99 ; p<0.01)$, but also significantly worse than detectability in the $250 \mathrm{~ms}$ blank condition $(t(6)=4.1 ; p<0.01)$. Finally, detectability of the target displacements in the two-bar condition, where the target disappears during the blanking interval, is only about $4 \%$ worse than detectability with the blanked target but no other stimuli in the field. This difference is not significant $(p>0.05)$. The results show that, in order to induce the 'blanking' effect, the screen has not necessarily to be blank. Rather, the critical feature of the blanking effect seems to be the temporary absence of the (localized) target. But is it critical that the postsaccadic object is identical to the presaccadic target?

\section{Experiment 2: target substitution}

Experiment 1 studied the possible effect of amodal completion on space constancy and the blanking ef- 
fect. In this experiment, the postsaccadic occluder (the bar) was neither localized in the horizontal dimension nor did target and occluder share featural properties. In Experiment 2 we examined the effect of presenting a well-localized spatial pattern, visually very dissimilar to the target, during the blanking interval. If the postsaccadic object must be geometrically similar to the original target in order to be accepted as a spatial reference, the visual system should simply ignore the stimulus. However, if the space constancy system accepts a highly dissimilar alternative pattern as a 'place holder' for the reference object, the location of this pattern should strongly affect displacement perception of the target.

\section{Methods}

Six naive subjects participated in this experiment. Again, four different conditions were included in the experiment. Typical sequences of stimulus presentations are given in Fig. 2A. As in the previous experiment, there was a 'No blank' and a '250 ms blank' condition, without presentation of any other stimuli (Fig. 2A, first and second columns), In the critical conditions, additional stimuli were presented. Note that concerning the target, the stimuli were identical to the ' $250 \mathrm{~ms}$ blank' condition. During the blanking interval a pattern appeared consisting of two rectangles, $0.4^{\circ}$ wide $\times 0.56^{\circ}$ high. They were $0.94^{\circ}$ apart, positioned vertically above and below the eventual position of the post-blanking target.

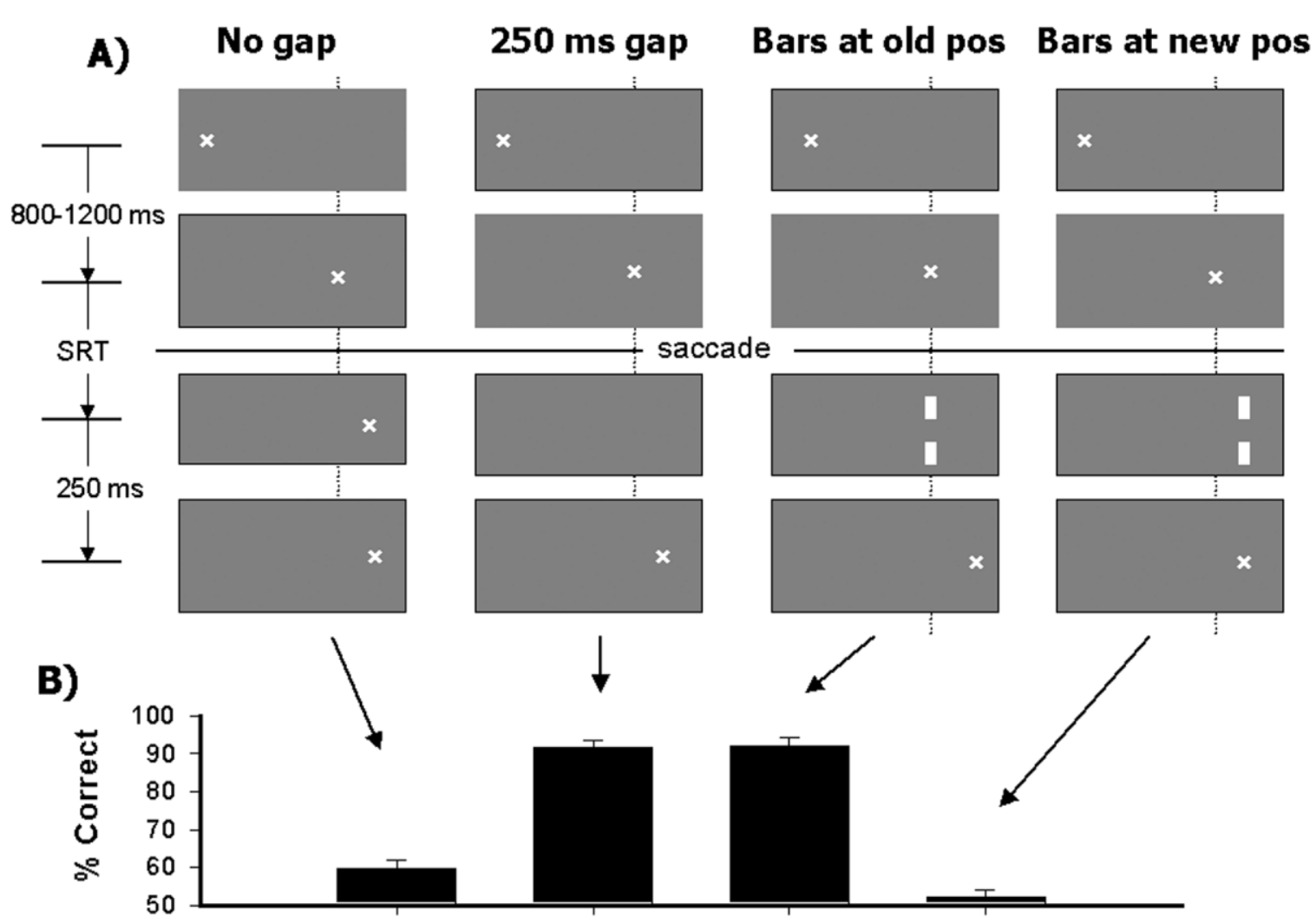

Fig. 2. (A) Stimulus conditions in Experiment 2. Display format as in Fig. 1. The two leftmost columns again represent the 'no blank' and the ' $250 \mathrm{~ms}$ blank' control conditions. In the third condition, two short rectangles appear for $250 \mathrm{~ms}$ at the horizontal (presaccadic) location of the blanked target. In the fourth condition, the bars are presented at the new (displaced) position of the blanked target. (B) Discrimination performance in control conditions and with presentation of a pattern of two short rectangles at the old and at the new target position during the blanking interval. 
Thus the two rectangles replaced the original target for $250 \mathrm{~ms}$ postsaccadically. The rectangles did not overlap the target spatially, but their horizontal position was well-defined. At the same time, the empty region in the centre of the substituted stimulus clearly revealed that the original saccade target had disappeared, and there were no common elements between the original target and the substituted target. Concerning the location of the substitute target, two different conditions were applied. In a first condition, the rectangles appeared at the location of the old (presaccadic) target (Fig. 2A, third column). In a second condition, the rectangles appeared at the location where the displaced target would appear after the blanking period (Fig. 2A, rightmost column).

If the absence of visual information about target position alone is sufficient to yield the detectability of target displacement, discrimination performance should be independent of the rectangle pattern presentation, and should be as good as in the blanking condition. If the rectangle pattern present immediately after the saccade is accepted as a spatial reference, however, the perception of target displacement should be determined by the pattern location in a well-predictable way: in the condition where the pattern appears at the old target position, displacement detection for the saccade target should be perfect. When the pattern appears at the new target position, however, no displacement should be perceived because the short rectangles specify the future position of the target as the new spatial reference.

\section{Results}

Discrimination performance in this experiment is presented in Fig. 2B. While performance is at chance for the 'No blank' control condition, displacement detection is close to perfect for the ' $250 \mathrm{~ms}$ blank' condition, reproducing the blanking effect. The more interesting cases however are the situations in which the additional rectangles are presented. These stimuli are irrelevant for the subject's task, and the sequence of presentation of the target is identical to the 'blank' condition. Nevertheless, the location of the rectangle pattern now determined whether the subject perceived stability or displacement of the target. When the pattern of rectangles appeared at the presaccadic target location, discrimination of the target jumps was perfect, whereas discrimination with the pattern appearing at the new, displaced target location was similar to the non-blanking condition. Thus, in the latter case, the pattern did not enable the subjects to do any better than they had done in the no-blanking condition; they behaved as though the target had always been present. Thus, the results demonstrate that any localized object found at saccade end is taken as a spatial reference, even if obviously different from the target.

\section{Experiment 3: perception of transsaccadic form changes}

The two previous experiments demonstrated (1) that the absence of a target immediately after the saccade improves displacement detection and (2) that the postsaccadic presence of any localized stimulus, even if clearly not identical to the target, affects the localization of the target across the saccade. Experiment 3 investigates whether similar manipulations lead to similar effects if the detection of form changes rather than location is probed. With this experiment we intended to investigate, first, whether a temporal blanking of the stimulus immediately after the saccade also subserves the perception of transsaccadic changes of form, second, whether an occluding bar also acts like a blanking, and third, whether an irrelevant stimulus (a mask) present for a short period of time after saccade end leads to a deterioration of performance. The stimuli we used consisted of a rectangular, checkerboard-like array of small bright squares. We call these stimuli 'Phillips-patterns', since Phillips (1974) and Phillips and Christie (1977) introduced patterns of this kind for the study of visual short-term memory.

\section{Methods}

Four naive subjects participated in the experiment. The four different sequences of stimulus presentations are depicted in Fig. 3A. The subject initially fixated the fixation cross. After a random delay, a 'Phillips-pattern' appeared, 6 or $8^{\circ}$, to the left or to the right of fixation. This pattern was composed of an array of dark and bright small rectangles, each $0.27^{\circ}$ wide. As to the complexity of the pattern, two different levels of difficulty were used. In the 
A) No blank $250 \mathrm{~ms}$ blank $250 \mathrm{~ms}$ bar 250 ms mask

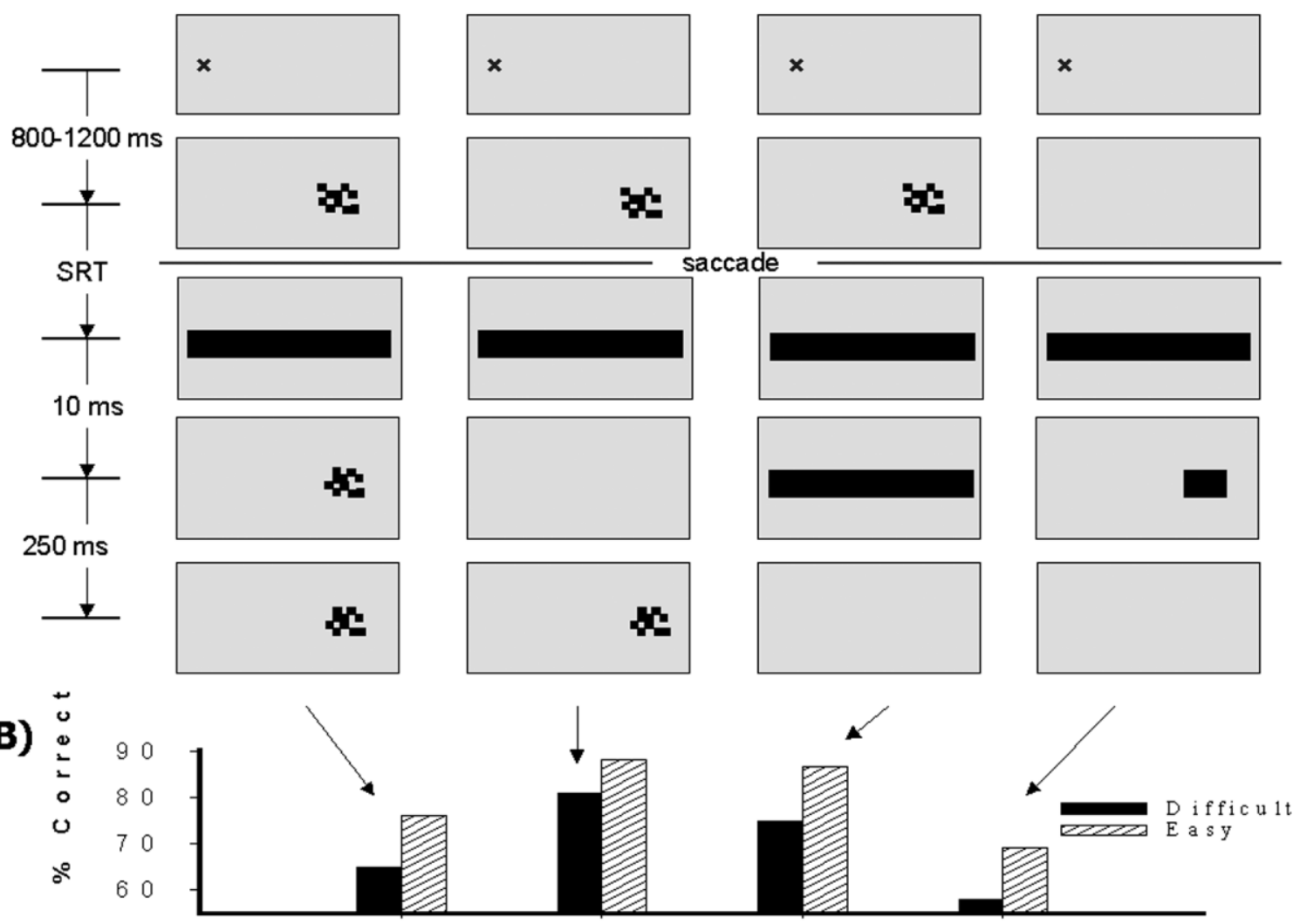

Fig. 3. (A) Stimulus conditions in Experiment 3. Display format as in Fig. 1. Here, subjects are asked to indicate, in a 2 AFC task, whether the upper or the lower half of the postsaccadic pattern was different from the presaccadic target pattern. (B) Percent correct discrimination for the four experimental conditions. Both levels of difficulty are presented separately ('Easy': light bars; 'Difficult': dark bars).

'Easy' condition the pattern was 4 squares high and 4 squares wide. In the 'Difficult' condition, the pattern was 4 squares high and 5 squares wide (see Fig. 3A). The subject was instructed to saccade to the appearing pattern. Triggered by the onset of the saccade, in all four conditions a bright horizontal bar covering the width of the display appeared for $10 \mathrm{~ms}$ (1 frame). This bar served to wipe-out all remains of phosphor persistence.

Again four different stimulus conditions were used. In the first (non-blanked) condition, the bar was replaced, still during the saccade, by a slightly changed version of the presaccadic pattern. In this postsaccadic pattern, one of the squares constituting the pattern had changed location. This change occurred, in $50 \%$ of the trials, in the upper half of the pattern, in the other $50 \%$ of the trials, in the lower half of the pattern. The subject had to indicate, in a $2 \mathrm{AFC}$ task, whether the change had occurred in the upper or the lower part of the pattern. The second 'blanked' condition was similar to the first except that a blank period of $250 \mathrm{~ms}$ was introduced, starting during the saccade. In the third condition, a long horizontal bright bar was presented for 250 ms that completely covered the stimulus area ('bar' condition). Only then did the changed pattern appear. Finally, in the forth condition ('mask' condition), the presaccadic pattern was replaced, for $250 \mathrm{~ms}$, by a homogeneous, bright rectangle covering the same area as the presaccadic target pattern, before the changed pattern appeared. 


\section{Results}

Fig. 3B depicts the percentages of correct discrimination for the four different conditions, plotted separately for the 'Easy' (light bars) and the 'Difficult' (dark bars) conditions. As a general data pattern the results show, not surprisingly, that the 'Easy' conditions lead to a better performance than the 'Difficult' conditions $(F(1,4)=108.6 ; p<0.001)$. More interestingly, however, the different experimental conditions also lead to significantly different performance $(F(3,12)=14.3 ; p<0.001)$. We tested this by posthoc pairwise comparisons (Bonferroni correction). As a first interesting result, the data reveal that the introduction of a $250 \mathrm{~ms}$ blank leads to a significant improvement of performance with respect to the no-blank control condition $(p=0.029)$. As for displacement detection, perception of transsaccadic changes is improved substantially by taking away target information at the end of the saccade, i.e., by a postsaccadic blank. The $250 \mathrm{~ms}$ 'bar' condition is not different from the 'blank' condition. Even if the target is replaced by a high-contrast (non-localized) bar, this is very helpful for transsaccadic discrimination performance. This implies that the essential aspect for the blanking effect is the absence of a localized stimulus. Most interesting is the result from the 'mask' case. Here, the visual system finds, when the saccade lands, a homogeneous stimulus of the same size as the presaccadic pattern. Under this condition, performance drops to a level that is as low as, if not worse than, the 'no-blank' condition (comparisons with the 'blank' and the 'bar' conditions yield highly significant differences, $p<0.001$ ). This suggests that the postsaccadic mask replaces the memory representation of the presaccadic stimulus, but only if the postsaccadic stimulus is a localized, object-like pattern.

\section{General discussion}

Postsaccadic visual information affects the use of transsaccadic memory

This study represents a continuation of our previous work on the blanking effect. The central result of our previous investigations was that when a saccade target is blanked even for a short interval during and after a saccade, its transsaccadic displacement becomes much more visible than when the target is continuously present (Deubel et al., 1996). A second important finding was that the object that is found by the visual system immediately after the saccade is normally perceived as stable, and it is taken as a spatial reference for judging whether other (blanked) objects had moved (Deubel et al., 1998).

The main objective of Experiment 1 was to investigate whether the postsaccadic presentation of a non-localized stimulus occluding the target area can produce an enhancement of displacement detection. The data of Fig. 1 confirm that when the saccade target is absent for a short temporal interval after the primary saccade, even small displacements that go otherwise undetected become obvious to the observer. This blanking effect is present when only the target disappears for a few hundred $\mathrm{ms}$, but it can also be elicited when the target area is covered by an occluding stimulus. The difference in performance between the 'Occluding bar' condition and the ' 250 ms blank' condition means that the beneficial effects of the blanking interval are not completely transferred when the target is occluded, as though the occluded 'target' was still able to influence the space constancy system to suspend the search for the reference object. Thus amodal presence of the target, even in the absence of any information about its current position, is sufficient to elicit some of the benefit to space constancy that normally occurs across saccades.

The results of the second experiment help to clarify one of our previous results (Deubel et al., 1998). In that paper, we found that the reference object need not be the saccade target, but could be another nearby pattern under some conditions. We presented two geometrically dissimilar targets, one the saccade goal and the other a distractor. If they were displaced relative to one another during a saccade, and one object had a blank, the visual system nearly always accepted the continuously present object as the reference object and perceived it to have remained at the same spatial position during the saccade. This was true whether the new reference object had originally been defined as the saccade goal or the distractor. Whether that object had actually been displaced during the saccade was irrelevant to this assigning of stability, and whether the newly 
accepted stable reference object was geometrically similar to the original saccade goal was also irrelevant. In our Experiment 2 we now asked for the effect of substituting the target, for a short interval after the saccade, with an otherwise irrelevant, but well localized pattern. It turned out that the continuous availability of information about object position in this condition determined whether the target was seen as stable or as jumping, even though the bar pattern was very different from the target. This finding is consistent with the assumption that the location of the short rectangles, present when the eyes land after the primary saccade, are taken by the visual system as the position of the (presaccadic) target. Obviously, the system detecting target position after the end of a saccade is not particularly selective about the geometric characteristics of the target. Visual form-related features of the reference object are unimportant in searching for the postsaccadic pattern, as long as the location is specified. As long as something appears in about the right place at the right time, according to our hypothesis the space constancy algorithm is satisfied that the reference object has been found, and no further computations are performed; the intrasaccadic displacement of the pattern goes unnoticed.

We conclude from this combination of results that the location of the target forms a major image feature used by the visual system in order to establish space constancy. If one of two objects is blanked, as was the case in our earlier experiments, then only the other object is available to become the reference object. Since the system is not selective about visual features, this distractor becomes the reference object by default, provided the location of the distractor is sufficiently close to the saccade goal object. By the time the blanked target reappears, the system is already committed to the other object as the reference object. The blanked target is then seen as displaced because its position is judged relative to the reference object, whose position is assumed to be stable.

Taken together, the present findings, as well as our previous results with the blanking effect (Deubel et al., 1996, 1998), support the idea that the visual system normally assumes that objects have not been displaced during saccadic eye movements, unless it is overwhelmed with contrary evidence, hence the high threshold for detecting simple image displacements during saccades. The present data can be interpreted in terms of the theories of visual stability described previously. The blanking effect demonstrates the importance of the stimuli that are present immediately after a saccade. According to our theoretical interpretation, the visual system searches for the presaccadic object of attention within a spatiotemporal 'constancy window', comparing the stored features with the new image around the saccade goal immediately after a saccade (Deubel et al., 1984; Irwin et al., 1994). If this target is found within a certain spatial and temporal window, the visual system assumes it to have remained stable during the saccade, and the target becomes a 'reference object' to determine the positions of other objects and textures (Deubel et al., 1996; McConkie and Currie, 1996). According to this theory, then, extraretinal signals are not used for transsaccadic integration under normal circumstances, because the reference object usually is found. The constancy mechanism concentrates on the region near the saccade target, with only secondary influence from other locations. Only changes in the saccade goal and possibly a few other attended objects are transferred accurately across saccades (Irwin et al., 1994). In other words, the structure of the postsaccadic visual scene plays a key role in the process of re-establishing visual direction following a saccade.

If the target is not found (as with target blanking), the assumption of stationarity is broken. Both extraretinal signals, such as efference copy and proprioception, and retinal signals from the visual context are used to compute the new target location. Only in this case does the system use information about sensory conditions before the saccade, ultimately leading to the detection of transsaccadic object displacements. This presaccadic information is stored across the saccade, but normally, when an object is present at the moment the primary saccade lands, it is discarded as soon as the reference object is found.

The tendency to discard, or substitute, the presaccadic information with the postsaccadic visual reafference is also obvious in the results from our Experiment 3 where we studied the effect of postsaccadic information on transsaccadic detection of form changes. Amazingly, the visual systems seems to obey very similar rules in this task, as it does for 
the task of displacement detection. First, our data demonstrate that postsaccadic blanking, as well as the presentation of a non-localized bar covering the target area leads to a strong improvement of transsaccadic perception of form change. This implies that more (form) information is contained in transsaccadic memory than normal (non-blank) tasks would suggest. Second, the condition with the postsaccadic mask shows that stimuli found after the saccade tend to replace the memory information, making the perception of transsaccadic changes very difficult. However, this effect requires that the postsaccadic stimulus is an object-like, localized item.

The present experiments also provide some characterization of the timing of the transsaccadic integration process. The results suggest that the presence or absence of an object at the moment when the eye lands is an essential determining factor for that object to become a spatial reference. This implies that the reference object need not be the saccade target: another nearby object can take that role, if the saccade target is blanked in a critical postsaccadic period so that it is unavailable for establishing a new calibration. This demonstrates that temporal continuity of an object is more important even than selection as a saccade target in establishing a reference object.

Our results necessitate some modification of the reference object theory that we described earlier (Deubel et al., 1996; McConkie and Currie, 1996). The visual system need not be committed to a single identified reference object before the saccade begins, for a task-irrelevant non-target object can become the reference object, and the system does not know in advance which object will be appropriate as the reference object. Whether an object is defined as target or distractor before the saccade seems to play little role in the postsaccadic determination of the reference object. Nevertheless, there is some independent evidence that the saccade goal target may be more important than other objects for postsaccadic visual calibration. Bischof and Kramer (1968), for instance, found perceived locations to be corrected more quickly near the saccadic goal than at other retinal positions. In a saccadic suppression experiment in which either the saccade target or another visual object such as the previous fixation target moved during the saccade, Heywood and Churcher (1981) showed that subjects often misattribute an intrasaccadic displacement of the saccade goal to a displacement of the other object, tending to preserve space constancy preferentially for the saccade goal. Finally, an important role of the saccade target is suggested by Ross et al. (1997), demonstrating that stimuli flashed shortly before a saccade are mislocalized such that they are perceived closer to the saccade target. Whether this 'spatial attraction' by the saccade target is related to the effect of our 'reference object' mechanism that tries to anchor presaccadically attended objects on the target found after the saccade must be clarified by further research (Lappe et al., 2000).

\section{Possible physiological mechanisms of visual stability across saccadic eye movements}

The mechanisms proposed above are based on the assumption that presaccadic information is remapped over the saccade in order to interact with the postsaccadic visual reafference. Neurons in lateral intraparietal cortex (LIP) described by Duhamel et al. (1992) may be performing some of the computations required by our theory. Receptive fields in this area shift to compensate for a saccade about $80 \mathrm{~ms}$ before the start of the movement. Thus the LIP seems to store presaccadic, visual information across the saccades and possesses quantitative spatial information about the saccade. The receptive fields are large, however, and would not be able to hold details of the features of a reference objects. Similar properties have been reported from neurons in the superior colliculus (Walker et al., 1995).

Further evidence for transsaccadic storage of saccade target features come from a recent study by Moore et al. (1998). They studied the visual selectivity of saccade-related responses of area V4 neurons in monkeys making delayed eye movements to receptive field stimuli of varying orientation. The neurons exhibit a selective presaccadic enhancement, quite separate from the response to the stimulus onset. The presaccadic enhancement appears to provide a strengthening of a decaying featural representation immediately before an eye movement is directed to visual targets. The authors suggest that this reactivation provides a mechanism by which a clear perception of the saccade goal can be maintained during the execution of the saccade, possibly for the 
purpose of establishing perceptual continuity across eye movements.

\section{Acknowledgements}

This study was supported by the Deutsche Forschungsgemeinschaft (SFB 462)

\section{References}

Irwin, 1991. Not in reference list.

Bischof, N. and Kramer, E. (1968) Untersuchungen und Überlegungen zur Richtungswahrnehmung bei willkürlichen sakkadischen Augenbewegungen. Psychol. Forsch., 32: 185218.

Breitmeyer, B.G. (1984) Visual Masking: An Interactive Approach. Oxford University Press, New York, NY.

Bridgeman, B. and Macknik, S.L. (1995) Saccadic suppression relies on luminance information. Psychol. Res., 58: 163-168.

Bridgeman, B., Hendry, D. and Stark, L. (1975) Failure to detect displacement of the visual world during saccadic eye movements. Vision Res., 15: 719-722.

Bridgeman, B., van der Heijden, A.H.C. and Velichkovsky, B.M. (1994) A theory of visual stability across saccadic eye movements. Behav. Brain Sci., 17: 247-292.

Burr, D.C., Morrone, M.C. and Ross, J. (1994) Selective suppression of the magnocellular visual pathway during saccadic eye movements. Nature, 371: 511-513.

Carlson-Radvansky, L.A. (1999) Memory for relational information across saccadic eye movements. Percept. Psychophys., 61: 919-934.

Crane, H.D. and Steele, C.M. (1985) Generation V dualPurkinje-image eye-tracker. Appl. Opt., 24: 527-537.

Currie, C.B., McConkie, G.W., Carlson-Radvansky, L.A. and Irwin, D.E. (2000) The role of the saccade target object in the perception of a visually stable world. Percept. Psychophys., 62: 673-683.

De Graef, P. and Verfaillie, K. (2001) Special issue: Transsaccadic object perception. Psychol. Belg., 41: 1-114.

De Graef, P. and Verfaillie, K. (2002) Commentary: Transsaccadic memory for visual object detail. In: J. Hyönä, D.P. Munoz, W. Heide and R. Radach (Eds.), The Brain's Eye: Neurobiological and Clinical Aspects of Oculomotor Research. Progress in Brain Research, Vol. 140, Elsevier, Amsterdam, pp. 000-000 (this volume).

Deubel, H. and Schneider, W.X. (1994) Can man bridge a gap? Behav. Brain Sci., 17: 259-260.

Deubel, H. and Schneider, W.X. (1996) Saccade target selection and object recognition: Evidence for a common attentional mechanism. Vision Res., 36: 1827-1837.

Deubel, H., Wolf, W. and Hauske, G. (1984) The evaluation of the oculomotor error signal. In: A.G. Gale and F. Johnson (Eds.), Theoretical and Applied Aspects of Eye Movement Research. Elsevier-North-Holland, Amsterdam, pp. 55-62.

Deubel, H., Schneider, W.X. and Bridgeman, B. (1996) Postsac- cadic target blanking prevents saccadic suppression of image displacement. Vision Res., 36: 985-996.

Deubel, H., Bridgeman, B. and Schneider, W.X. (1998) Immediate post-saccadic information mediates space constancy. Vision Res., 38: 3147-3159.

Duhamel, J.R., Colby, C. and Goldberg, M. (1992) The updating of the representation of visual space in parietal cortex by intended eye movements. Science, 225: 90-92.

Grüsser, O.-J., Krizic, A. and Weiss, L.-R. (1987) Afterimage movement during saccades in the dark. Vision Res., 27: 215226.

Helmholtz, H.v. (1963) Handbuch der Physiologischen Optik (1866). Dover.

Heywood, S. and Churcher, J. (1981) Direction-specific and position-specific effects upon detection of displacements during saccadic eye movements. Vision Res., 21: 255-261.

Hoffman, J.E. and Subramaniam, B. (1995) The role of visual attention in saccadic eye movements. Percept. Psychophys., 57: 787-795.

Honda, H. (1989) Perceptual localization of visual-stimuli flashed during saccades. Percept. Psychophys., 45: 162-174.

Honda, H. (1999) Modification of saccade-contingent visual mislocalization by the presence of a visual frame of reference. Vision Res., 39: 51-57.

Irwin, D.E. (1983) Evidence against visual integration across saccadic eye movements. Percept. Psychophys., 34: 49-57.

Irwin, D.E. (1992) Memory for position and identity across eye movements. J. Exp. Psychol. Learn. Mem. Cogn., 18: $307-$ 317.

Irwin, D.E. (1993a) Perceiving an integrated visual world. In: S.K.D.E. Meyer (Ed.), Attention and Performance XIV: Synergies in Experimental Psychology. MIT Press, Cambridge, MA, pp. 121-142.

Irwin, D.E. (1993b) Memory for spatial position across saccadic eye movements. In: G. d'Ydewalle and J. van Rensbergen (Eds.), Perception and Cognition: Advances in Eye Movement Research. North Holland, Amsterdam, pp. 323-332.

Irwin, D.E. and Gordon, R.D. (1998) Eye movements, attention and trans-saccadic memory. Vis. Cognit., 5: 127-155.

Irwin, D.E., McConkie, G.W., Carlson-Radvansky, L.A. and Currie, C. (1994) A localist evaluation solution for visual stability across saccades. Behav. Brain Sci., 17: 265-266.

Jonides, J., Irwin, D.E. and Yantis, S. (1982) Integrating visual information from successive fixations. Science, 215: 192-194.

Kowler, E., Anderson, E., Dosher, B. and Blaser, E. (1995) The role of attention in the programming of saccades. Vision Res., 35: 1897-1916.

Lappe, M., Awater, H. and Krekelberg, B. (2000) Postsaccadic visual references generate presaccadic compression of space. Nature, 403: 892-895.

Matin, L. (1972) Eye movements and perceived visual direction. In: D. Jameson and L. Hurvitch (Eds.), Handbook of Sensory Physiology 7. Springer, Berlin, pp. 331-380.

Matin, E. (1974) Saccadic suppression: A review and an analysis. Psychol. Bull., 81: 899-917.

McConkie, G.W. and Currie, C.B. (1996) Visual-stability across 
saccades while viewing complex pictures. J. Exp. Psychol. Hum. Percept. Perform., 22: 563-581.

McConkie, G.W. and Rayner, K. (1976) Identifying the span of the effective stimulus in reading: literature review and theories of reading. In: H. Singer and R. Ruddell (Eds.), Theoretical Models and Processes of Reading. International Reading Institute, Newark, DE, 2nd ed., pp. 137-162.

McConkie, G.W. and Zola, D. (1979) Is visual information integrated across successive fixations in reading?. Percept. Psychophys., 25: 221-224.

Michotte, A. (1963) The Perception of Causality. (T.R. Miles and C. Miles, Transl.) Methuen, London.

Moore, T., Tolias, A.S. and Schiller, P.H. (1998) Visual representations during saccadic eye movements. Proc. Natl. Acad. Sci. USA, 95: 8981-8984.

O'Regan, J.K. and Levy-Schoen, A. (1983) Integrating visual information from successive fixations: Does trans-saccadic fusion exist?. Vision Res., 23: 765-768.

Phillips, W.A. (1974) On the distinction between sensory storage and short-term visual memory. Percept. Psychophys., 16: 283290.

Phillips, W.A. and Christie, D. (1977) Interference with visualization. Q. J. Exp. Psychol., 29: 637-650.

Pollatsek, A., Rayner, K. and Collins, W.E. (1984) Integrating pictorial information across eye movements. J. Exp. Psychol. Gen., 113: 426-442.

Rayner, K. (1998) Eye movements in reading and information processing - 20 years of research. Psychol. Bull., 124: 372422.

Rayner, K. and Pollatsek, A. (1983) Is visual information integrated across saccades?. Percept. Psychophys., 34: 39-48.

Rayner, K., McConkie, G.W. and Zola, D. (1980) Integrating information across eye movements. Cognit. Psychol., 12: 206226.

Rensink, R.A. (2002) Changes. In: J. Hyönä, D.P. Munoz, W. Heide and R. Radach (Eds.), The Brain's Eye: Neurobiological and Clinical Aspects of Oculomotor Research. Progress in Brain Research, Vol. 140, Elsevier, Amsterdam, pp. 000-000 (this volume).

Rensink, R.A., O'Regan, J.K. and Clark, J.J. (1997) To see or not to see: The need for attention to perceive changes in scenes. Psychol. Sci., 8: 368-373.

Ross, J., Morrone, M.C. and Burr, D.C. (1997) Compression of visual space before saccades. Nature, 386: 598-601.

Ross, J., Morrone, M.C., Goldberg, M.E. and Burr, D.C. (2001) Changes in visual perception at the time of saccades. Trends Neurosci., 24: 113-121.

Schlag, J. and Schlag-Rey, M. (1995) Illusory localization of stimuli flashed in the dark before saccades. Vision Res., 35: 2347-2357.

Schneider, W.X. (1999) Visual-spatial working memory, attention and scene representation: A neuro-cognitive theory. Psychol. Res., 62: 220-236.

Sperry, R.W. (1950) Neural basis of the spontaneous optokinetic response produced by visual inversion. J. Comp. Physiol. Psychol., 43: 482-489.

Verfaillie, K. and De Graef, P. (2000) Transsaccadic memory for position and orientation of saccade source and target. J. Exp. Psychol. Hum. Percept. Perform., 26: 1243-1259.

Von Holst, E. and Mittelstaedt, H. (1954) Das Reafferenzprinzip. Naturwissenschaften, 37: 464-476.

Walker, M.F., Fitzgibbon, E.J. and Goldberg, M.E. (1995) Neurons in the monkey superior colliculus predict the visual result of impending saccadic eye-movements. J. Neurophysiol., 73: 1988-2003.

Wolf, W., Hauske, G. and Lupp, U. (1978) How presaccadic gratings modify postsaccadic modulation transfer function. Vision Res., 18: 1173-1179.

Wolf, W., Hauske, G. and Lupp, U. (1980) Interaction of pre- and postsaccadic patterns having the same coordinates is space. Vision Res., 20: 117-124. 